\title{
Developing Behavior Change Interventions
}

\section{Hankonen, Nelli Elisa}

Cambridge University Press

2020-07

Hankonen, N E \& Hardeman , W 2020 , Developing Behavior Change Interventions . in M S Hagger , L D Cameron, K Hamilton, N Hankonen \& T Lintunen (eds), The Handbook of Behavior Change . , 21, Cambridge Handbooks in Psychology , Cambridge University Press , pp. 300-317 . https://doi.org/10.1017/9781108677318.021

http://hdl.handle.net/10138/327016

https://doi.org/10.1017/9781108677318.021

unspecified

publishedVersion

Downloaded from Helda, University of Helsinki institutional repository.

This is an electronic reprint of the original article.

This reprint may differ from the original in pagination and typographic detail.

Please cite the original version. 


\title{
21 Developing Behavior Change Interventions
}

\author{
Nelli Hankonen and Wendy Hardeman
}

\section{Practical Summary}

When trying to help people change behavior, it is important that intervention designers consider carefully what actions might bring about the desired outcomes and why. A systematic approach to intervention development aids this process. Key tasks are (1) identify and analyze the problem addressed by the intervention; (2) identify how the intervention will achieve the desired outcomes, decide on its content and delivery mode (s), and design a logic model or program theory; (3) develop intervention materials or prototypes (e.g., interface); and (4) test or pilot the intervention iteratively in an early stage. This approach can be adopted to develop new interventions and to optimize existing interventions. It is useful to build an explicit model of assumed influences of an intervention during its design, including influences on behavior, contextual influences on implementation, and the causal pathway involved. The decisions about intervention content and delivery modes are informed by an understanding of the target group, behaviors, context, and working mechanisms of behavior change techniques.

\subsection{Introduction}

Intervention development is an essential process in which several parties work together to produce an intervention that is fit-for-purpose and likely to be effective in changing the behavior of the target population. Parties involved are likely to include practitioners, researchers, the target group, and other stakeholders (see also Chapters 24 and 25, this volume). The scope of development work is influenced by the available time and resources. The aim of this chapter is to provide an overview of the basic steps in the intervention development process. The chapter shows how careful development helps ensure that the intervention is (1) informed by evidence from multiple sources (e.g., the research literature, the target group or population, stakeholders, relevant theory); (2) that the intervention is an appropriate solution to a problem; (3) that context and any challenges in the implementation of the intervention have been carefully considered and addressed; (4) that feasibility, acceptability, and resource use (value for money) have been considered at an early stage; (5) that the intervention is optimized prior to evaluation; and (6) that the evaluation will focus on key uncertainties about the intervention. A carefully reported development process is also crucial in understanding issues arising from intervention implementation, such as providing insight into what may have gone wrong if the intervention does not achieve stipulated goals or is labeled as "ineffective." It also provides necessary detail to contribute to the evidence base of behavioral interventions and behavior change theories. The chapter will focus on the development of behavior change interventions across disciplines and

https://doi.org/10.1017/9781108677318.021 
behavioral domains and cover interventions that focus on changing the behavior of individuals as well as larger-scale interventions that change the behavior of groups all the way up to populationlevel interventions.

Key frameworks that have been applied to inform development of behavior change interventions are the intervention mapping approach (Bartholomew Eldredge et al., 2016), the behavior change wheel (Michie, Atkins, \& West, 2014; Michie, van Stralen, \& West, 2011), and the UK Medical Research Council (MRC) framework for complex interventions. Readers are directed to these key frameworks for further reading and for examples of the application of the various development steps (for a comprehensive list, the reader is directed to Araújo-Soares et al., 2018; O'Cathain et al., 2019; Chapter 19, this volume). The steps suggested by the frameworks are shown in Table 21.1. Intervention mapping explains a careful, stepwise process in translating theories of behavior change (see the chapters in Part I) into practical health promotion programs. The behavior change wheel (Michie et al., 2011; Michie et al., 2014) integrates theory and evidence in informing decisions about intervention design together with stakeholders. It is based on a comprehensive review and synthesis of existing frameworks for intervention development. By contrast, the UK Medical Research Council (MRC) framework (Craig et al., 2008) offers more general guidance to intervention development, focusing on identifying the evidence base, identifying or developing theory, and modeling process and outcomes.

\subsection{Overarching Principles in Developing Behavior Change Interventions}

This section describes some key general principles relevant to the tasks involved in intervention development (see Chapters 19 and 20, this volume). Commonly used frameworks during the development of behavior change interventions share the following key tasks (see Table 21.1):

Task 1: Identifying and analyzing the problem addressed in behavioral terms and developing intervention objectives.

Task 2: Identifying intervention mechanisms, content, and delivery mode, including the design of a logic model or program theory.

Task 3: Developing materials and/or technology.

Task 4: Early, iterative testing of the intervention and empirical optimization.

In research, these phases are usually followed by a feasibility or pilot study, a substantive evaluation study (e.g., a randomized controlled trial), and an implementation phase (see Chapter 22, this volume). The following sections describe some overarching principles that apply to all four tasks.

\subsubsection{Flexibility: An Iterative Rather Than Linear Process}

Although the key tasks involved in intervention development are presented in a specific order, intervention development should be considered an iterative rather than linear process. For example, pretesting of an intervention (task 4) may reveal the need to revise intervention materials (task 3). The above steps are applicable to developing new interventions and to optimizing existing interventions. For example, an intervention in the real world may fail to reach its intended target group and is, thus, in need of optimization. If the intervention has not been specified or described well, development of a logic model (task 2) may help reveal weak links that could be optimized, in this example an asset-based approach might be adopted, which brings the intervention to the target population using existing resources (e.g., community groups). Alternatively, the tasks may be completed by different stakeholders, for example practitioners and decision makers may have done a problem analysis and a multidisciplinary 


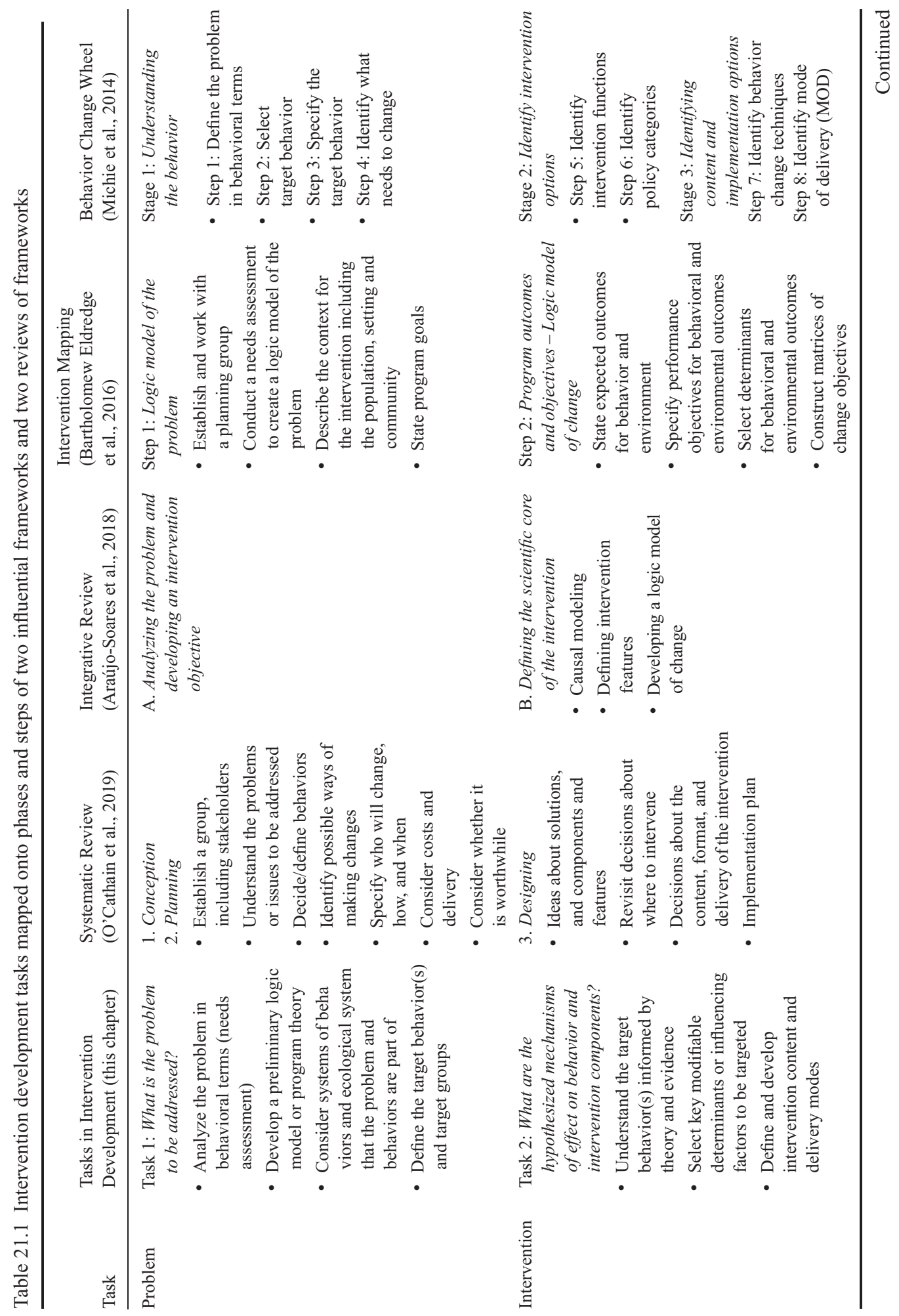



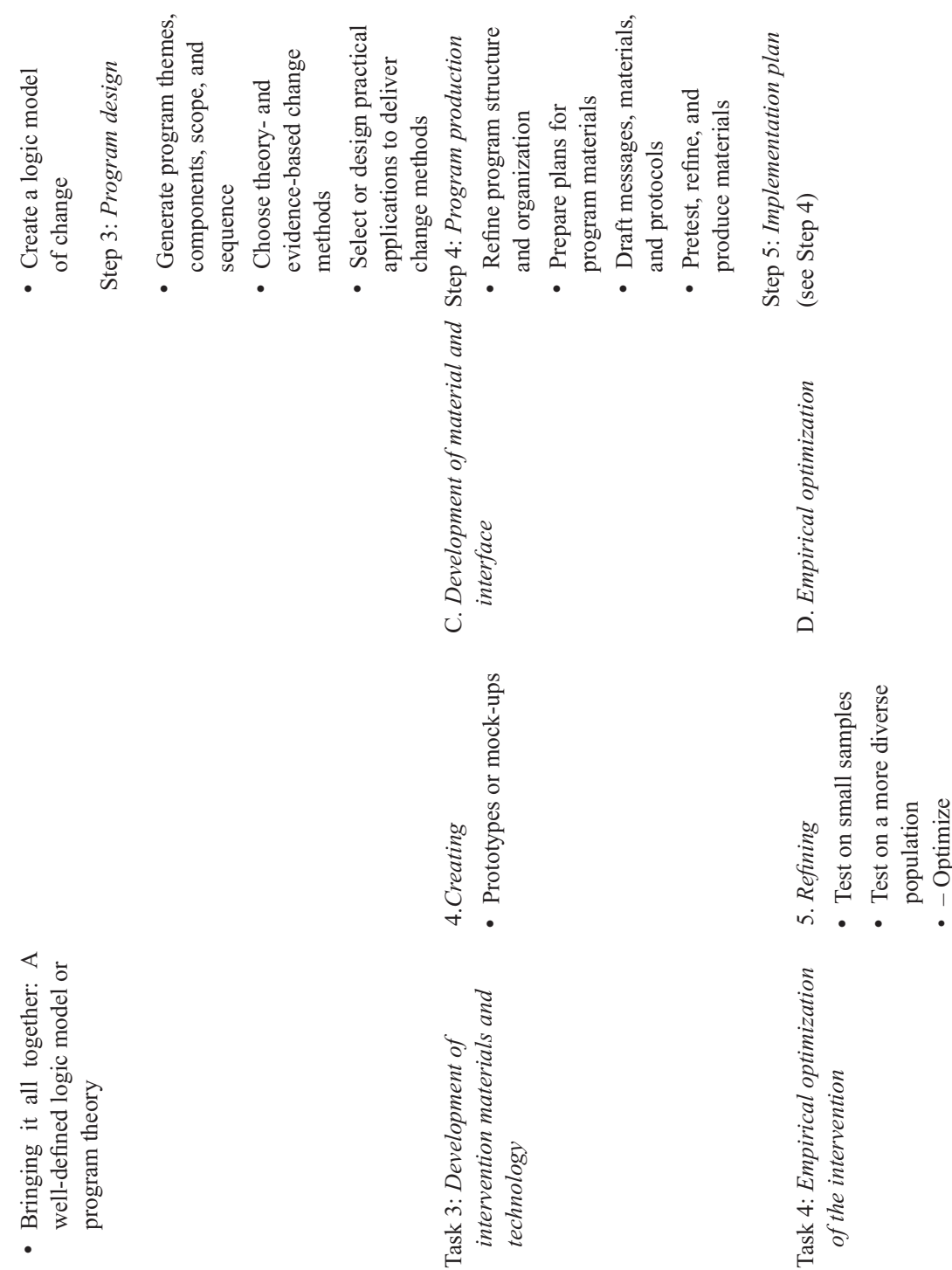

$\frac{\sqrt[n]{\pi}}{\sum_{\overline{0}}^{\frac{\pi}{\pi}}}$

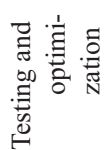




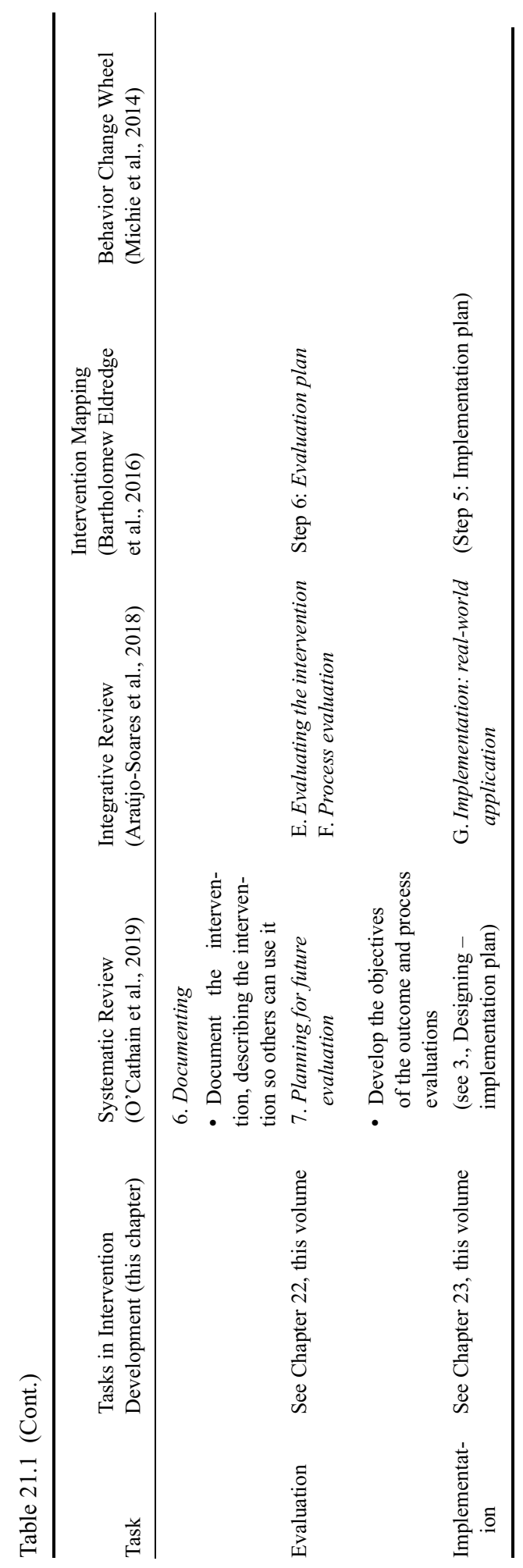


team may do the remaining tasks for the intervention development.

The key tasks may differ across interventions according to (1) the context of intervention development, that is, whether the intervention is in a research, policy, or practice context, and (2) the mode of delivery. Focusing on context, the time frame and scope of intervention development work need to be matched to resources, expertise, and time. Intervention development as a part of a research study is often more comprehensive, based on theory and evidence, and takes longer than when this is done by real-world organizations (e.g., a health protection agency that needs to act quickly in response to emerging issues such as a new flu outbreak). A lengthy, comprehensive, empirically based intervention development is rarely the case in practice. With time and resource constraints, one may conduct rapid reviews of the evidence and a quick succession of stakeholder consultations, rather than systematic reviews and comprehensive qualitative research, and focus on key uncertainties surrounding the intervention. Reporting the process carefully (see Sidebar 21.1) is useful for any intervention to inform long-term monitoring, audit, or evaluation.

\subsubsection{Consider Constraints in Intervention Development}

Intervention developers rarely have all the options for intervention development available. Commissioners or funders often set constraints in terms of a specific mode of delivery (e.g., a mobile phone application), context, (e.g., school-based intervention), or providers (e.g., teachers or nurses). A priori practical constraints may dictate maximum dose (e.g., maximum feasible contact time with target group of five minutes for a health care consultation or three hours in school curriculum) or a cost threshold for the intervention. Any constraints need to be made explicit as they influence decisions at all stages (see also Sidebar 21.1) and may limit the available candidate intervention components. All constraints need to be monitored throughout the intervention development. Common constraints are costs. Economic modeling and estimation of resource use during intervention development can help ensure that the resulting intervention is likely to be cost-effective, and many intervention development teams now include economists who build an economic model to test assumptions (see Chapter 26, this volume).

Time and financial resources may also affect the scope of intervention development work, especially for interventions developed or optimized in the real world. In these instances, it can be helpful to draw a logic model and/or to define the key uncertainties about the proposed intervention and focus limited development resources on addressing these uncertainties.

\subsubsection{Including Stakeholders and End Users in the Intervention Development Team}

Across all tasks, intervention development should be informed by relevant expertise and evidence. Academic disciplines may include psychology, policy, sociology, economics, business, computer science, and service design. Key stakeholders could include members of the public, representatives of the target group(s), intervention providers/professionals, commissioners, policy makers, and funders. Involvement of experts in the relevant disciplines and key stakeholders aids understanding of the intervention context and informs strategic decisions that reflect scientific and practical expertise, experiential evidence, and the preferences and views of the end users and those whose involvement is critical for the adoption and implementation of the intervention (e.g., Bartholomew Eldredge et al., 2016). It also assists with the recruitment and engagement of the target group and the cost-effectiveness, coownership, and codesign of a comprehensive 
logic model or program theory. Ideally, key stakeholders should be consulted in the earliest stages of the initial problem formulation (see Chapters 24 and 25, this volume). The selection of stakeholder groups is influenced by the skills needed to develop the intervention, those involved in the adoption and implementation of the intervention, commissioning the intervention, and those who will help increase impact. For example, decision makers who can ensure implementation of the behavioral intervention in the long term should ideally be included in the intervention development team. Their involvement will facilitate reach, adoption, and implementation of the intervention once the research evaluating the intervention has been concluded (Chapter 23 , this volume).

\subsubsection{Using a "Complex Systems" Approach During Intervention Development}

Recently, calls have been made to develop and study interventions with explicit complexity science perspective (e.g., Hawe, 2015; Heino et al., 2019; Skivington et al., 2018). The target setting and behavior of an intervention, as well as the intervention itself, can be considered a complex, adaptive, and dynamic system, which is more than the sum of its parts (see Gomersall, 2018; Resnicow \& Page, 2008). Complex systems share, for example, the following key features: (1) interconnections in the system, that is, relationships and interconnections between different parts or components are important rather than the individual parts separately; (2) selforganization and emergence, that is, order is created in a system without explicit hierarchical direction or central planning; and (3) coevolution of the system and the environment, that is, the system (e.g., intervention) and the environment influence each other's development (for an overview, see Heino et al., 2019). There is some evidence that interventions that make use of these features are more effective than more simplistic interventions (Leykum et al., 2007). Although many interventions and policies incorporate some principles of the complex systems approach, traditional intervention development frameworks have not explicitly drawn on these fully. Systems thinking could be more explicitly integrated and adopted in both such frameworks and behavior change theories. Additionally, this approach can encourage awareness of real-world uncertainties that the proposed intervention could address or consider. Therefore, intervention developers are encouraged not only to consider how the intervention is expected to work (i.e., the internal intervention logic and complexity therein) but to consider the overall system, what parts of the system could influence the intervention, and how the intervention could lead to wider system change.

\subsection{Key Tasks in Intervention Development}

The following sections report key tasks in intervention development that help to iteratively identify, develop, and refine the content and mode(s) of delivery of a theory- and evidence-based intervention, including consideration of context, implementation, and hypothesized mechanism of effect (see Table 21.1).

Documenting the sequence of decisions during intervention development can be helpful. The important background features and decisions involved may include, but are not limited to describing, (1) issues at the beginning (e.g., preparatory work to describe the team and planned development process); (2) the time used and available for intervention development process (e.g., length of design period, frequency of design meetings); (3) the possible commissioner demands, limitations, or requests of the intervention or the development process (e.g., future use, use of technology, limited financial resources, quick timeline for development); and 
(4) details of decisions during the process, including considered alternative options, leading to choices about the intervention (e.g., program components/activities; intermediate targets; behavior change techniques to target predictors/mechanisms, including to what extent various combinations of techniques were explicitly considered and left out; see also Sidebar 21.1).

\subsubsection{Task 1: What Is the Problem to Be Addressed?}

A new intervention is essentially a solution to a perceived and defined problem. If the problem is not well defined, the intervention may not achieve the desired outcomes, such as increasing patient safety, reducing costs in the health service, improving literary levels, or reducing inequalities.

Therefore, the first step is to clearly define the problem to be addressed by the intervention and justify why the problem matters. The problem may be defined at several levels (e.g., individual, organizational, and societal). Examples of problems are the burden on society, such as sickness absences from work or demands on health care services due to the rising prevalence of Type 2 diabetes (societal-level problems); health care professionals lacking the time for promoting healthy behaviors (an organizational-level problem); or parents deciding not to vaccinate their children (an individual-level problem). The importance of a health problem is often quantified as its cost to health and social care systems and society and the burden of disease for patients. It is important to identify and define the target population at an early stage: who is affected by the problem; who might benefit most from the proposed intervention; how they can be reached by the intervention; and how these considerations influence choices about the content and mode of delivery of the intervention. Again, this involves a careful review of context and evidence, including research, policy documents, and stakeholder consultations. The next sections outline some of the specific tasks to be completed during the definition of the problem to be addressed.

\subsubsection{Analyze the Problem in Behavioral Terms}

A thorough analysis of the problem includes an understanding of the context of the proposed or existing intervention. Identification of the current behavioral status of the target population (e.g., are people doing what they should be doing to achieve desired outcomes?) and understanding and defining what needs to change, who needs to change, and when and where the change might occur are all important questions to address. This analysis will inform the objectives or goals of the intervention. Specifying these also allows a detailed understanding of potential constraints, linked to context (e.g., school), intervention duration (e.g., five minutes maximum), or cost (e.g., cost per participant). It helps avoid inappropriate and ineffective solutions to the problem. As an example, when the delivery of evidence-based health care is suboptimal (e.g., hospital nurses do not wash their hands in between seeing patients or family doctors overprescribe antibiotics), common interventions are guidelines and training. These tend to focus on the individual level, whereas a detailed problem analysis might have revealed that the problem is not at the individual level but at the organizational or team level (e.g., lack of alcohol rub, incentivization of care, perceived team roles).

In intervention mapping, needs assessment involves assessing the problem (e.g., air pollution in inner cities) and its likely behavioral, social, and environmental causes. This involves the identification and definition of the sequence of behaviors needed to achieve desired outcomes, based on existing evidence (e.g., people using more sustainable alternatives, such as public transport, cycling, or walking rather than driving a car; city councils building the infrastructure to promote sustainable travel) and the identification 
Guidance such as TIDieR (Hoffmann et al., 2014) has improved the transparent reporting of interventions. Furthermore, the process of and decisions during intervention development can be reported. For example, Araújo-Soares et al. (2018) suggest reporting several items in their step 2, "defining the scientific core of the intervention," which corresponds to the task 2 of this chapter. The checklist (adapted) includes the following:

(1) Understand causal/contextual factors (causal modeling)

Describe:

a. formal (behavioral) theories used in understanding the predictors of the target behavior

b. how key uncertainties were identified to select the aim(s) of evidence synthesis

c. literature search and review process

d. the rationale/aims and the process of (potential) original empirical research

e. rating of influencing factors (psychological, social, predictors/mechanisms) for changeability and relevance

(2) Develop a logic model or program theory

Describe:

a. the process of developing the model (if possible, include early and later versions)

b. key explicit criteria (e.g., acceptability, cost-effectiveness) in making decisions about the model

c. whether and which other similar existing interventions were used in developing the logic model or program theory or whether an existing intervention was used as core basis and retrofitted

d. key uncertainties left in the logic model or program theory and the possible "weak links" the development team considers merit further investigation

e. assessment of evaluability potential of the intervention

f. (and develop) a dark logic model that describes considerations made around potential unintended consequences and steps made to avoid them

(3) Define intervention features

Describe:

a. decision processes (including considered alternative options) leading to decisions about

i. program components/activities

ii. intermediate targets

iii. behavior change techniques or methods to target predictors/mechanisms, e.g., to what extent various combinations of techniques were explicitly considered and left out

iv. dose/intensity/frequency/duration

v. delivery channel(s)

vi. providers (expertise/background/training)

vii. location/infrastructure

b. whether and how anticipated acceptability was investigated

c. the decision processes related to scope for local adaptation and extent of fidelity assessment 
of intermediate outcomes relevant for the hypothesized mechanisms of the intervention (Bartholomew Eldredge et al., 2016).

\subsubsection{Develop a Preliminary Logic Model or Program Theory}

A clearly defined problem to be addressed by the proposed intervention and specification of the intervention's context provides a helpful starting point for an early draft of a logic model or program theory. A logic model is a graphical depiction of "if-then" relationships between the resources needed for the intervention, the activities or components of the intervention, and the hypothesized short-term, medium-term, and long-term outcomes and impact of the intervention (W. K. Kellogg Foundation, 2004). Logic models focus on the big ideas, not the details of your intervention, and are depicted on one page. A logic model reads like a series of "if-then" statements that connect the components of the intervention. Logic models vary in content. A logic model facilitates a shared understanding among key stakeholders about the problem addressed, the justification for the intervention, its components, and how it is hypothesized to work. It can help identify weak links in need of development work to resolve. Different templates for logic models are available (University of Wisconsin, 2008; University of Wisconsin-Extension, 2003; W. K. Kellogg Foundation, 2004). Readers are directed to various examples of logic models and program theories (Davidoff et al., 2015; Evans et al., 2018; Morgan et al., 2015; Tully et al., 2019; Appendix 21.1, supplemental materials).

The literature uses multiple terms - logic models, program theories, intervention theories, and theories of change - and consensus is lacking about their similarities and differences. Logic models tend to depict a temporal sequence, describing inputs/ resources, outputs (activities and participation), outcomes, and impact, whereas program theories and theories of change tend to depict how the intervention is expected to work in its context. However, the distinctions are blurred: The W. K. Kellogg Foundation (2004) states the theory approach as one of the three approaches to their logic models: the theory of change that influenced intervention design and plan. Davidoff and colleagues (2015) report that a program theory specifies (1) the components, expected outcomes, and the methods for assessing the outcomes of an intervention, often in the form of a logic model, and (2) the "hypotheses" of the intervention, that is, specification of the rationale behind, and underlying assumptions of, the mechanisms that link the processes and inputs of the intended and unintended outcomes of the intervention, as well as the conditions and context necessary for effectiveness (Davidoff et al., 2015). Realist program theories consist of context, mechanism, and outcome configurations (CMOCs), which are generated in consultation with stakeholders and allow one to understand "what works for whom in what circumstances" (Pawson, 2006).

A pragmatic approach can be recommended: In selecting a suitable format, for example logic model or program theory, consider which format fits the proposed intervention and purpose best, for instance who will use the logic model or program theory, how will it be used, and whether it is used during the development, implementation, or evaluation phase. If the key purpose is to tell the "big story" of the intervention and its effects over time to present to a wide range of stakeholders, or details of implementation, then a logic model may be best. If the key purpose is to depict the causal pathways, then a program theory may be suitable. If the purpose is to depict the problem, one could use the intervention mapping guidance. Finally, one can combine components of logic models and program theories (e.g., describe the problem, intervention activities, and causal pathways over time).

In the later tasks, the logic model or program theory can be extended to include determinants of the target behavior or behaviors, the behavior change techniques that will be used to change them (e.g., goal setting and incentives), other 
intervention components (e.g., such as a face-toface meetings, leaflets, or a smartphone app), and measures of outcomes and impact in the short, medium, and long term. The task of the development team is then to refine this initial logic model, together with stakeholders. It should be noted that a logic model of the problem can be separated from the logic model of the proposed solution (see Bartholomew Eldredge et al., 2016) or combined.

\subsubsection{Consider Systems of Behaviors and an Ecological System of the Target Problem and/or Behavior}

Problems addressed and behaviors targeted by a proposed intervention do not exist in a vacuum but are dependent on other behaviors and contexts, that is, they are part of a wider system. Other behaviors may facilitate the target behavior or conflict with it. Therefore, intervention developers need to consider the target behavior in the context of other behaviors (e.g., clustering of unhealthy behaviors and consumer behaviors). Behavior results from a system of influences, including proximal individual cognitive and emotional factors, social and community influences, and distal factors such as living and working conditions (environment, housing, education) and socioeconomic, cultural, and environmental conditions (see Chapters 17, 18, and 28, this volume). Further, creating a map of social agents (which groups of people may influence the problem and/or the target group's behavior) can be helpful. Consideration of the functionalities of the system, including interactions and feedback loops, is also important.

\subsubsection{Defining the Target Behavior(s) and Target Group(s)}

Influential frameworks for intervention development stress the importance of clearly defining and specifying the target behavior(s) for the intervention. The selection of the target behavior depends on various considerations, such as being an important cause of the problem, modifiability, and acceptability by the target group. For example, in an intervention study to reduce sedentary behavior among adolescents, empirical studies showed that an early selection of "reducing screen time" as the target behavior was perceived as highly unacceptable by the target group. As a consequence, the target behavior was changed to reducing sitting time in the school context (Hankonen et al., 2016). Once the target behavior has been identified, it can be added to the logic model along with the intervention objectives. Also, at this point it is important to check that the definition of the target groups is clear and agreed on, as well as possible subpopulations/segments.

\subsubsection{Task 2: What Are the Hypothesized Mechanisms of Effect on Behavior and Intervention Components?}

In this phase, developers will further populate the logic model by including the hypothesized mechanisms of effect, informed by an understanding of the behavior, and intervention components to the logic model. The developers will need to source information or evidence on key components, or "links in the chain," of the proposed mechanism (i.e., how the intervention will achieve its effect).

The team decides on and records:

- Key modifiable influences of the target behavior(s)

- Hypothesized causal mechanisms of intervention effects

- The nature of the intervention (intervention function)

- Behavior change technique(s)

- Mode(s) of delivery, e.g., face-to-face or digital

- Intervention provider(s) (if relevant)

- Personalization and tailoring of intervention content (especially relevant for digital interventions)

- Setting(s)

- Intervention intensity, such as timing and dose 
- Strategies to optimize reach, (cost-)effectiveness, adoption, implementation, and long-term maintenance of the intervention

This is not an exhaustive list as the key issues that need to be considered depend on the nature of the intervention and its context.

\subsubsection{Understand the Target Behavior(s) Informed by Theory and Evidence}

A wide range of theories and frameworks have been applied to predict and understand behavior (for examples, see the chapters in Part I of this handbook). The capability, opportunity, motivation - behavior (COM-B) model can be used as a starting point (Michie et al., 2011), as it is an overarching framework of behavior, as well as the theoretical domains framework as a framework to achieve a more fine-grained understanding of behavioral influences.

Understanding the behavior also involves a consideration of preparatory behaviors, which may be influenced by unique determinants. For example, for someone to take medication as prescribed, they will need to obtain the medication from a pharmacy or, in order to use a condom, both partners need to negotiate its use. As a result, behavioral targets may extend beyond the single behavioral outcome of the intervention (see Section 21.1.3.5). Preparatory behaviors can be added to the logic model along with intervention objectives.

\subsubsection{Select Key Modifiable Determinants to Be Targeted}

Target behaviors and determinants of behavior (or influences on behavior) targeted by the proposed intervention need to be prioritized and selected. Appendix 21.2 (supplemental materials) provides a list of criteria that are commonly used in intervention development: acceptability, practicability, effectiveness/relevance, affordability, possible side effects, and equity. A key consideration is changeability: the extent that determinants can be changed based on current evidence and theory and the impact of those changes on key outcomes (e.g., Araújo-Soares et al., 2018; Bartholomew Eldredge et al., 2016). Changeable, modifiable factors that have a strong relationship to the target behavior are potential targets for interventions (e.g., Michie et al., 2011).

Identifying the key modifiable influences on behavior often requires a range of quantitative and qualitative methods, data, and sources (see Appendixes 21.3 and 21.4, supplemental materials).

\subsubsection{Define and Develop Intervention Content and Delivery Modes}

Once the key modifiable determinants of the target behavior have been identified, the next task is to select intervention techniques, that is, the methods or strategies that will affect a change in behavior by changing the identified determinants such as cognitions or environmental variables. This selection should be based on formative evidence on the effectiveness of the techniques in changing the determinants and the subsequent change in the target behavior or the plausibility of such links, based on evidence from pathways of similar interventions, if such evidence is not available.

Often behavior change interventions have had heterogeneous effects, which can make it challenging to select intervention components. In this case, it may be possible to retrospectively "code" the behavior change techniques and other intervention features (e.g., modes of delivery) used in previous intervention work during evidence synthesis as part of the development work in order to provide the requisite evidence on which to base the selection of determinants and matched techniques. Such an approach would enable the intervention designer to explore, narratively or quantitatively, the extent to which intervention techniques and other features are associated with intervention effectiveness (e.g., Dombrowski et al., 2012). This approach is not without its limitations - for example, studies rarely report 
the fidelity of delivery of the techniques as well as other features and whether participants used them in their daily lives ("enactment"), both of which impact intervention effectiveness (see Toomey et al., 2019).

Other relevant sources of evidence to inform the selection of intervention components include evidence regarding the setting, target behavior, behavior change techniques, and mode of delivery. This evidence can come from evidence synthesis or existing reviews, qualitative research, and consultation with stakeholders (see Appendix 21.4, supplemental materials).

Acceptability is a further important factor that should be accounted for when selecting techniques and other intervention features (e.g., modes of delivery) for inclusion in an intervention. Acceptability of techniques and other intervention features, as perceived by those delivering or receiving the intervention, is defined as a "multifaceted construct that reflects the extent to which people delivering or receiving a healthcare intervention consider it to be appropriate, based on anticipated or experienced cognitive and emotional responses to the intervention" (Sekhon, Cartwright, \& Francis, 2017, p. 4). Perceptions about acceptability are linked to ethicality, perceived effectiveness, likability, burden, and coherence of the intervention. Acceptability of intervention components necessitates consultation with stakeholders as part of the coproduction of the intervention, because feedback from those groups is pivotal for the adaptation of the intervention content to the specific context and target group or population. Empirically, acceptability can be investigated before the intervention takes place through discussion with the appropriate stakeholders, who report on the anticipated acceptability of an intervention scenario, but should also be part of the evaluation of the intervention during its delivery or retrospectively after the intervention is complete during follow-up measurement (Sekhon et al., 2017; see Chapter 22, this volume).
Finally, other criteria often need to be taken into consideration. Stakeholders or commissioners of the intervention may have strong preferences about certain features such as the mode of delivery. For example, the team developing the intervention may have been commissioned by an organization to develop an intervention delivered via a preferred mode of delivery. For instance, the organization may specify that an intervention aiming to change the behavior of employees or children in a school setting should be delivered by a mobile phone app or a web-based platform. In addition, feasibility considerations play an important role in the selection of intervention content. For example, the duration or intensity of an intervention can be constrained by its context, and cost-effectiveness considerations may set upper limits on intervention costs. For example, if the selected behavior change techniques to be used in a planned intervention have tended to be delivered via a face-to-face, practitioner-client mode of delivery, the costs associated with the practitioner delivery need to be taken into account in feasibility considerations and it may be decided that such costs are prohibitive, necessitating a rethink of the mode of delivery and, perhaps as a consequence, the techniques selected. For instance, a feasibility study into five-minute physical activity advice in primary care evaluated action planning, an evidence-based BCT. However, no participant defined an action plan, so it did not prove feasible in clinical practice (Pears et al., 2015).

\subsubsection{Bringing It All Together: $A$ Well- Defined Logic Model or Program Theory}

Section 21.3.1.2 introduced logic models as a graphic representation of the problem, intervention components, causal pathways, process and outcome measures, and expected impact. It is recommended that a logic model or program theory is developed in an iterative manner, starting with an initial draft that includes the context and 
the problem. The model or theory is then populated once decisions are made about how the intervention is expected to achieve its outcomes (causal pathways), about its behavior change techniques, and about the modes of delivery. A carefully constructed logic model provides intervention designers, intervention deliverers, and all stakeholders with a clear "visual map" of the proposed intervention (Appendix 21.1, supplemental materials). The presumed causal mechanisms can also be expressed verbally, for example, in a series of "if -then," "so that" statements (Davidoff et al., 2015) or as intervention mapping matrices (Bartholomew Eldredge et al., 2016).

It is important to specify not only the desired outcomes and impact of the intervention but also any unintended, negative, and harmful consequences. A "dark" logic model refers to a careful elaboration of potential pathways by which the intervention could lead to negative or harmful consequences (Bonell et al., 2015), such as increasing health inequalities (see Chapter 27, this volume). Instead of only identifying potential harms, this process clearly outlines the mechanisms through which such harms may take place - for example, people who are most in need of the intervention are not reached due to recruitment challenges or a lack of resources (e.g., travel expenses and childcare) required to take part in the intervention (for an example, see Cook et al., 2018).

\subsubsection{Task 3: Development of Intervention Materials and Technology}

If a planned intervention is not attractive and easy to use, the target group will not try it out or continue to use it. The intervention designer wants to maximize the reach and engagement of the target population but for behavioral interventions delivered via digital modes (see Chapter 29, this volume), for example, engagement is a key challenge (Perski et al., 2017). Promoting engagement of key stakeholders with the intervention is another critical design task (see Chapter 24, this volume). Design decisions on the "look" and "feel" of intervention materials, such as posters or leaflets, depend on the target audience, behavior, and the chosen mode of delivery. Successful engagement with the intervention relies on effective coproducing of the intervention with stakeholders, particularly with members of the target group. Adopting a multidisciplinary approach when selecting the team employed to design the intervention is also important to enable input from design-related and creative disciplines such as computer science, environmental design, or educational sciences. Final program material production (e.g., posters, videos, smartphone apps) may involve creative consultants, artists, or graphic designers.

It can be beneficial to work with advertising professionals, graphical artists, and website and app developers in the production of intervention materials and technology. However, it is important to bear in mind that approaches to changing consumer preferences for products are different to changing other behaviors, and interventions with a host of features may not necessarily increase engagement. Researchers, the target group, practitioners, and any other stakeholders need to work closely with those designing the intervention materials. Writing design documents to guide the creation and review of intervention materials and technology can help in ensuring that behavioral science insights and intervention strategies are adequately transferred into the production of materials (Bartholomew Eldredge et al., 2016). The importance of behavior change expertise input is particularly important to note, as advertising professionals may have limited knowledge and experience of the techniques used to change behavior and their effectiveness (see Chapters 19 and 20, this volume).

To make the intervention attractive, clear, and relevant to end users, it is good practice to engage 
them at an early stage (see Chapters 24 and 25, this volume) using consensus conferences, codesign workshops, and user-centered design (O'Cathain et al., 2019). It is crucial to coproduce early prototypes of intervention materials with the end users and conduct early user testing in an iterative manner (agile development). In practice, this task often happens in parallel with task 2 .

\subsubsection{Task 4: Empirical Optimization of the Intervention}

In this task, the intervention designer pilot tests the intervention in a small group of end users from the target group or population of interest in order to identify and solve any problems or issues before a feasibility study or, in real-world situations, to conduct a full-scale pilot test of the intervention. This can involve initial testing using research methods such as a small-scale experiment followed up with surveys, interviews (e.g., data-prompted interviews; Kwasnicka et al., 2015), or focus groups with the participants to collect evidence on issues such as feasibility, acceptability, and fidelity. The feedback may help refine the intervention content and/or mode of delivery.

Formal feasibility testing of the intervention, in which the full intervention is tested prior to a fullscale evaluation, is common and can take many forms (see Chapter 22, this volume). Most intervention development frameworks recommend pilot or feasibility testing of a "beta" version of the intervention, in a small-scale study, prior to a wider-scale evaluation such as a fully powered study and rollout. A key aim is to provide important feedback on the intervention from the target population that will enhance its acceptability, feasibility, and fidelity. One approach to refining behavioral interventions is the multiphase optimization strategy (MOST), a framework to rigorously test and select the best options for intervention components (Collins, Murphy, \& Strecher, 2007).

\subsection{Implications for Research, Practice, and Translation}

The series of tasks required to design behavioral interventions can, and should, be addressed systematically and reported transparently, within the limits of resources. A detailed understanding of the problem, behavior, and context will facilitate the adoption and implementation of interventions. While acknowledging the complexity and dynamic nature of human and social systems including the design of behavior change interventions - a systematic approach to intervention development is important if the design team is to develop behavior change interventions that are feasible, acceptable, and, above all, optimally effective.

Numerous approaches and frameworks for the development of behavior change interventions share many common features. This chapter has identified those commonalities but there are also a number of unique features of each. It would be helpful to develop evidence-based guidance for researchers, practitioners, and policy makers on which approach is best suited to a particular problem, setting, and intervention. This would necessitate the systematic development of an evidence base in which different approaches were applied to intervention development in like contexts, behaviors, and populations. The generation of such evidence would be useful for all groups involved in intervention design but especially for those working in practice and policy. A further important advance would be to systematically review the content of different approaches to behavior change intervention design (e.g., O'Cathain et al., 2019), with a view to consolidate common features and identify and incorporate useful unique features in order to arrive at a comprehensive and optimally effective approach, perhaps via expert consensus. Another improvement would be the development of a decision tree that helps researchers and intervention developers in practice decide which framework is most applicable for their proposed intervention. 
Several key future developments and methodological challenges in intervention development methods can be identified. First, more focus is needed on developing interventions that can be adopted, implemented, and sustained in the "real world" through early testing of interventions/ components. Second, further work is needed to understand how to best harness, address, model, and plan for characteristics of dynamic complex systems in intervention development. Third, more transparent reporting of intervention development would help users and other intervention developers understand why some choices were made, in addition to just seeing the final intervention, and thereby improve insight in the "art" of intervention development. Sharing details of decisions will help advance the field by clarifying what alternative pathways developers usually choose between and why. This will also help in developing better methods to best adapt and optimize existing interventions.

\subsection{Summary and Conclusion}

This chapter has outlined the key tasks involved in developing interventions and general principles that underpin all the tasks. It has emphasized the iterative nature of intervention development, the importance of coproducing interventions with the target group and stakeholders, making use of theory and evidence, and the need to adapt the development process according to constraints.

\section{References}

Araújo-Soares, V., Hankonen, N., Presseau, J., Rodrigues, A., \& Sniehotta, F. F. (2018).

Developing behavior change interventions for selfmanagement in chronic illness: An integrative overview. European Psychologist, 24, 7-25. https://doi.org/10.1027/1016-9040/a000330

Bartholomew Eldredge, L. K., Markham, C. M., Ruiter, R. A. C., Fernández, M. E., Kok, G., \& Parcel, G.
S. (2016). Planning Health Promotion Programs: An Intervention Mapping Approach. Hoboken, NJ: John Wiley \& Sons.

Bonell, C., Jamal, F., Melendez-Torres, G. J., \& Cummins, S. (2015). "Dark logic": Theorising the harmful consequences of public health interventions. Journal of Epidemiology and Community Health, 69, 95-98. https://doi.org/ 10.1136/jech-2014-204671

Collins, L. M., Murphy, S. A., \& Strecher, V. (2007). The multiphase optimization strategy (MOST) and the sequential multiple assignment randomized trial (SMART): New methods for more potent eHealth interventions. American Journal of Preventive Medicine, 32, S112-S118. https://doi.org/10.1016/j.amepre.2007.01.022

Cook, P. A., Hargreaves, S. C., Burns, E. J. et al. (2018). Communities in charge of alcohol (CICA): A protocol for a stepped-wedge randomised control trial of an alcohol health champions programme. BMC Public Health, 18, 522. https://doi.org/ 10.1186/s12889-018-5410-0

Craig, P., Dieppe, P., Macintyre, S., Michie, S., Nazareth, I., \& Petticrew, M. (2008). Developing and evaluating complex interventions: The new Medical Research Council guidance. BMJ, 337, a1655. https://doi.org/10.1136/bmj.a1655

Davidoff, F., Dixon-Woods, M., Leviton, L., \& Michie, S. (2015). Demystifying theory and its use in improvement. BMJ Quality and Safety, 24, 228238. https://doi.org/10.1136/bmjqs-2014-003627

Dombrowski, S. U., Sniehotta, F. F., Avenell, A., Johnston, M., MacLennan, G., \& Araújo-Soares, V. (2012). Identifying active ingredients in complex behavioural interventions for obese adults with obesity-related co-morbidities or additional risk factors for co-morbidities: A systematic review. Health Psychology Review, 6, 7-32. https://doi.org/ 10.1080/17437199.2010.513298

Evans, R., Brockman, R., Grey, J. et al. (2018). A cluster randomised controlled trial of the Wellbeing in Secondary Education (WISE) Project: An intervention to improve the mental health support and training available to secondary school teachers: protocol for an integrated process evaluation. Trials, 19, 270. https://doi.org/ 10.1186/s13063-018-2617-4 
Gomersall, T. (2018). Complex adaptive systems: A new approach for understanding health practices. Health Psychology Review, 12, 405-418. https:// doi.org/10.1080/17437199.2018.1488603

Hankonen, N., Heino, M. T. J., Araújo-Soares, V. et al. (2016). "Let's Move It": A school-based multilevel intervention to increase physical activity and reduce sedentary behaviour among older adolescents in vocational secondary schools: A study protocol for a cluster-randomised trial. BMC Public Health, 16, 451. https://doi.org/ 10.1186/s12889-016-3094-x

Hawe, P. (2015). Lessons from complex interventions to improve health. Annual Review of Public Health, 36, 307-323. https://doi.org/10.1146/ annurev-publhealth-031912-114421

Heino, M. T. J., Noonan, C., Knittle, K., \& Hankonen, N. (2019). Studying behaviour change mechanisms under complexity. Unpublished manuscript, University of Helsinki.

Hoffmann, T. C., Glasziou, P. P., Boutron, I. et al. (2014). Better reporting of interventions: Template for intervention description and replication (TIDieR) checklist and guide. $B M J$, 348, g1687. https://doi.org/10.1136/bmj.g1687

Kwasnicka, D., Dombrowski, S. U., White, M., \& Sniehotta, F. F. (2015). Data-prompted interviews: Using individual ecological data to stimulate narratives and explore meanings. Health Psychology, 34, 1191-1194. https://doi.org/ 10.1037/hea0000234

Leykum, L. K., Pugh, J., Lawrence, V. et al. (2007). Organizational interventions employing principles of complexity science have improved outcomes for patients with Type II diabetes. Implementation Science, 2, 28. https://doi.org/ 10.1186/1748-5908-2-28

Michie, S., Atkins, L., \& West, R. (2014). The Behaviour Change Wheel: A Guide to Designing Interventions. London: Silverback Publishing.

Michie, S., van Stralen, M. M., \& West, R. (2011). The behaviour change wheel: A new method for characterising and designing behaviour change interventions. Implementation Science, 6, 42. https://doi.org/10.1186/1748-5908-6-42

Morgan, H., Hoddinott, P., Thomson, G. et al. (2015). Benefits of Incentives for Breastfeeding and
Smoking Cessation in Pregnancy (BIBS): A Mixed-Methods Study to Inform Trial Design. Southampton: NIHR Journals Library. O’Cathain, A., Croot, L., Sworn, K. et al. (2019). Taxonomy of approaches to developing interventions to improve health: A systematic methods overview. Pilot and Feasibility Studies, 5. https://doi.org/10.1186/s40814-019-0425-6

Pawson, R. (2006). Evidence-Based Policy: A Realist Perspective. London: SAGE.

Pears, S., Morton, K., Bijker, M., Sutton, S., Hardeman, W., \& VBI Programme Team. (2015).

Development and feasibility study of very brief interventions for physical activity in primary care. BMC Public Health, 15, 333. https://doi.org/ 10.1186/s12889-015-1703-8

Perski, O., Blandford, A., West, R., \& Michie, S. (2017). Conceptualising engagement with digital behaviour change interventions: A systematic review using principles from critical interpretive synthesis. Translational Behavioral Medicine, 7, 254-267. https://doi.org/10.1007/s13142-016-0453-1

Resnicow, K., \& Page, S. E. (2008). Embracing chaos and complexity: A quantum change for public health. American Journal of Public Health, 98, 1382-1389. https://doi.org/10.2105/AJPH .2007 .129460

Sekhon, M., Cartwright, M., \& Francis, J. (2017). Acceptability of healthcare interventions: An overview of reviews and development of a theoretical framework. BMC Health Services Research, 17, 88. https://doi.org/10.1186/s12913017-2031-8

Skivington, K., Matthews, L., Craig, P., Simpson, S., \& Moore, L. (2018). Developing and evaluating complex interventions: Updating Medical Research Council guidance to take account of new methodological and theoretical approaches. The Lancet, 392, S2. Meeting abstract: Public Health Science 2018, Belfast, Northern Ireland, November 23, 2018. http://dx.doi.org/10.1016/ s0140-6736(18)32865-4

Toomey, E., Hardeman, W., Hankonen, N. et al. (2019). Focusing on fidelity: Recommendations for improving intervention fidelity within trials of health behavioral interventions. Unpublished manuscript, National University of Ireland, Galway. 
Tully, M. A., Cunningham, C., Wright, A. et al. (2019). Peer-led walking programme to increase physical activity in inactive 60-to 70-year-olds: Walk with Me pilot RCT. Public Health Research, 7. https:// doi.org/10.3310/phr07100

University of Wisconsin. (2008). Developing a logic model: Teaching and training guide. Program Development and Evaluation. Website. https://fyi .extension.wisc.edu/programdevelopment/logicmodels/
University of Wisconsin-Extension. (2003). Enhancing program performance with logic models. Program Development and Evaluation. Website. https://fyi .extension.wisc.edu/programdevelopment/design ing-programs/

W. K. Kellogg Foundation. (2004). Using Logic Models to Bring Together Planning, Evaluation, and Action: Logic Model Development Guide. Michigan: W. K. Kellogg Foundation. 